\title{
Night on Earth: Mapping decadal changes of anthropogenic night light in Asia
}

\author{
Christopher Small ${ }^{\mathrm{a}, *}$, Christopher D. Elvidge ${ }^{\mathrm{b}}$ \\ a Lamont Doherty Earth Observatory, Columbia University, New York City, USA \\ b Earth Observation Group, NOAA National Geophysical Data Center, Boulder, CO, USA
}

\section{A R T I C L E I N F O}

\section{Article history:}

Received 8 July 2011

Accepted 24 February 2012

\section{Keywords:}

Urban

Night light

DMSP-OLS

Landsat

Zipf

Asia

India

China

Nightsat

\begin{abstract}
A B S T R A C T
The defense meteorological satellite program (DMSP) operational linescan system (OLS) sensors have imaged emitted light from Earth's surface since the 1970s. Temporal overlap in the missions of 5 OLS sensors allows for intercalibration of the annual composites over the past 19 years (Elvidge et al., 2009). The resulting image time series captures a spatiotemporal signature of the growth and evolution of lighted human settlements and development. We use empirical orthogonal function (EOF) analysis and the temporal feature space to characterize and quantify patterns of temporal change in stable night light brightness and spatial extent since 1992. Temporal EOF analysis provides a statistical basis for representing spatially abundant temporal patterns in the image time series as uncorrelated vectors of brightness as a function of time from 1992 to 2009. The variance partition of the eigenvalue spectrum combined with temporal structure of the EOFs and spatial structure of the PCs provides a basis for distinguishing between deterministic multi-year trends and stochastic year-to-year variance. The low order EOFs and principal components (PC) space together discriminate both earlier (1990s) and later (2000s) increases and decreases in brightness. Inverse transformation of these low order dimensions reduces stochastic variance sufficiently so that tri-temporal composites depict potentially deterministic decadal trends. The most pronounced changes occur in Asia. At critical brightness threshold we find an $18 \%$ increase in the number of spatially distinct lights and an $80 \%$ increase in lighted area in southern and eastern Asia between 1992 and 2009. During this time both China and India experienced a $\sim 20 \%$ increase in number of lights and a $\sim 270 \%$ increase in lighted area - although the timing of the increase is later in China than in India. Throughout Asia a variety of different patterns of brightness increase are apparent in tri-temporal brightness composites - as well as some conspicuous areas of apparently decreasing background luminance and, in many places, intermittent light suggesting development of infrastructure rather than persistently lighted development. Vicarious validation using higher resolution Landsat imagery verifies multiple phases of urban growth in several cities as well as the consistent presence of low DN (< 15) background luminance for many agricultural areas. Lights also allow us to quantify changes in the size distribution and connectedness of different intensities of development. Over a wide range of brightnesses, the size distributions of spatially contiguous lighted area are consistent with power laws with exponents near -1 as predicted by Zipf's Law for cities. However, the larger lighted segments are much larger than individual cities; they correspond to vast spatial networks of contiguous development (Small et al., 2011).
\end{abstract}

(c) 2012 Published by Elsevier B.V.

\section{Introduction}

The defense meteorological satellite program (DMSP) operational linescan system (OLS) has imaged emitted light from Earth's surface since the 1970s. In 1992 a digital archive was established for DMSP-OLS data at the NOAA National Geophysical Data Center. Annual composites of temporally stable night light have been processed and distributed by the NGDC for every year since 1992. Temporal overlap in the missions of multiple OLS sensors allows for

\footnotetext{
* Corresponding author.

E-mail address: small@LDEO.columbia.edu (C. Small).
}

intercalibration of the 30 annual composites over the past 19 years (Elvidge et al., 2009). These data offer the opportunity to quantify a unique spatiotemporal signature of human settlement growth and evolution. Here we summarize some initial results of a spatiotemporal analysis of annual change in the extent, distribution and intensity of anthropogenic night light in Asia.

The objective of this study is to quantify apparent changes in the number, extent and brightness of stable night lights in Asia. We limit the geographic scope of this study to Asia because this is where we observe the greatest changes. The intention is to illustrate a methodology for characterization and vicarious validation of apparent changes in areas where anthropogenic changes are large enough to be clearly distinguished from other types of change 
related to sensor calibration and atmospheric effects. The first part of the study illustrates the use of the principal component (PC) transformation and empirical orthogonal function (EOF) analysis with multi-temporal night light data. Background and details of the EOF analysis are given by Small (under review). Here we present the results of the EOF analysis and discuss the spatiotemporal changes quantified by the analysis. The second part of the study presents a vicarious validation of changes in night lights using changes in land cover as imaged by Landsats 5 and 7. The third part of the study presents spatial and temporal variations in the area, number and rank size distributions of lighted areas and discusses the implications for urban growth processes.

\section{Mapping night light from space}

Since 1994, NGDC has had an active program focused on global mapping of nighttime lights using the data collected by the DMSP-OLS sensors. The basic product is a global annual cloud-free composite, which averages the OLS visible band data for one satellite from the cloud-free segments of individual orbits. By compositing only the cloud-free segments the impact of atmospheric effects is minimized. Over the years, NGDC has developed automatic algorithms for screening the quality of the nighttime visible band observations to remove areas contaminated by sunlight, moonlight, and the presence of clouds (Baugh et al., 2009; Elvidge et al., 1997). For this study we used the NGDC annual stable lights product. In the stable lights annual cloud-free composite product generation, fires and other ephemeral lights are removed based on their high brightness and short duration. Background noise is removed by setting thresholds based on visible band values found in areas known to be free of detectable lights. In 2010, NGDC released the version 4 time series of Stable Lights, spanning the years 1992-2009. These are available online at http://www.ngdc.noaa.gov/dmsp/downloadV4composites.html. Because the OLS visible band has no on-board calibration system, the data from each satellite year should be intercalibrated prior to quantitative analysis of the time series. Baugh et al. (2009) provides a description of the algorithms used to make the v.4 stable lights products. The intercalibration procedure is described by Elvidge et al. (2009). This intercalibration is applied to the time series of annual composites used in our analysis.

Temporally stable night lights, as measured by the Defense Meteorological Satellite Program-Operational Line Scanner(DMSPOLS), provide a unique proxy for anthropogenic development. In previous studies brightness and spatial extent of emitted light has been correlated to population density (Briggs et al., 2007; Sutton et al., 2001), built area density (Elvidge et al., 2007), economic activity (Doll et al., 2006; Ghosh et al., 2010; Henderson et al., 2009), and electrification rates (Elvidge et al., submitted for publication). Night lights are known to overestimate the spatial extent of development at the periphery of settlements (Elvidge et al., 1997) but the spatial extent of this overglow is generally small compared to the total area of all but the smallest settlements (Small et al., 2005). Comparisons of stable light with $30 \mathrm{~m}$ resolution Landsat imagery on a wide variety of population density gradients indicates that average brightness increases with increasing spatial density of Shortwave Infrared reflectance and shadow associated with constructed surfaces (Small et al., 2005) as well as actual maps of impervious surfaces (Elvidge et al., 2007).

In this study, we use tri-temporal change maps to illustrate decadal changes in brightness and spatial extent of night lights. Displaying brightness of three annual average brightness composites in the red, green and blue channels of a color image highlights areas of change as color deviations from the gray shades that indicate equal brightnesses in all three years. A tri-temporal composite of 1994, 2001 and 2009 for southeastern Asia is shown in Fig. 1. At the scale of this figure some large area changes in background luminance are visible but most of the changes are too small to be seen. A linear stretch between 0 and 12 DN highlights changes in background luminance at the expense of saturating changes in brighter more developed areas. This figure illustrates two challenges of representing change with tri-temporal brightness composites: (1) showing change in both bright and dim areas simultaneously and (2) showing deterministic decadal changes in the presence of spurious year-to-year variability. In order to circumvent these challenges, and make better use of the information content of the composite time series, we present an approach that uses all 30 annual composites to quantify change in lighted areas. However, we retain the convenience of using tri-temporal color composites to illustrate these changes. We accomplish this by separating distinct components of spatiotemporal change using EOF analysis to represent the interannual trends of the observed changes in night light. As explained in detail below, the EOF analysis provides an estimate of the dimensionality of the image time series and a basis for separating deterministic and stochastic components of the spatiotemporal changes observed. In the context of this study, dimensionality refers to the different background levels and temporal patterns of change and their corresponding spatial distributions. We use the EOF analysis to minimize the effect of stochastic year-to-year variance in brightness thereby emphasizing interannual trends in the brightness and spatial extent of lighted area. The structure of the temporal feature space (explained below) also provides a concise representation of the overall distribution of changes in the night light data.

\section{Spatio-temporal dimensionality and empirical orthogonal function analysis}

Principal component transformations are commonly used to represent uncorrelated modes of variance in high dimensional data. Different types of PC transform are used to reduce the dimensionality of multispectral imagery (e.g. Green et al., 1988; Lee et al., 1990; Singh and Harrison, 1985) and to represent the topology of spectral feature spaces (Adams et al., 1986; Crist and Cicone, 1984; Johnson et al., 1985; Kauth and Thomas, 1976; Smith et al., 1985). Because spectral bands are often correlated, PC transforms provide an efficient low dimensional projection of the uncorrelated components of the spectral feature space. The same property applies to temporal dimensions. PC transforms have also been used to represent uncorrelated patterns in multi-temporal imagery (Richards, 1984) (Eastman and Fulk, 1993; Townshend et al., 1985) and for change detection (Byrne et al., 1980; Fung and LeDrew, 1987). In meteorology and oceanography the PC transformation provides the basis of empirical orthogonal function analysis; a standard tool for analysis of spatio-temporal patterns and processes (see Bretherton et al., 1992; Preisendorffer, 1988; von_Storch and Zwiers, 1999 for overviews).

The utility of the PC transform for representing spatiotemporal processes is related to the fact that any location-specific $(x)$ pixel time series $P_{x \mathrm{t}}$ contained in an $N$ image time series can be represented as a combination of temporal patterns and their location-specific components as:

$P_{x \mathrm{t}}=\sum_{i=1}^{N} C_{i x} F_{i \mathrm{t}}$

where $C_{i x}$ is the spatial principal component $(\mathrm{PC})$ and $F_{i t}$ is the corresponding temporal empirical orthogonal function (EOF) and $i$ is the dimension. The EOFs are the eigenvectors of the covariance matrix. EOFs represent uncorrelated temporal patterns of variability within the data. The PCs are the corresponding weights that represent the 


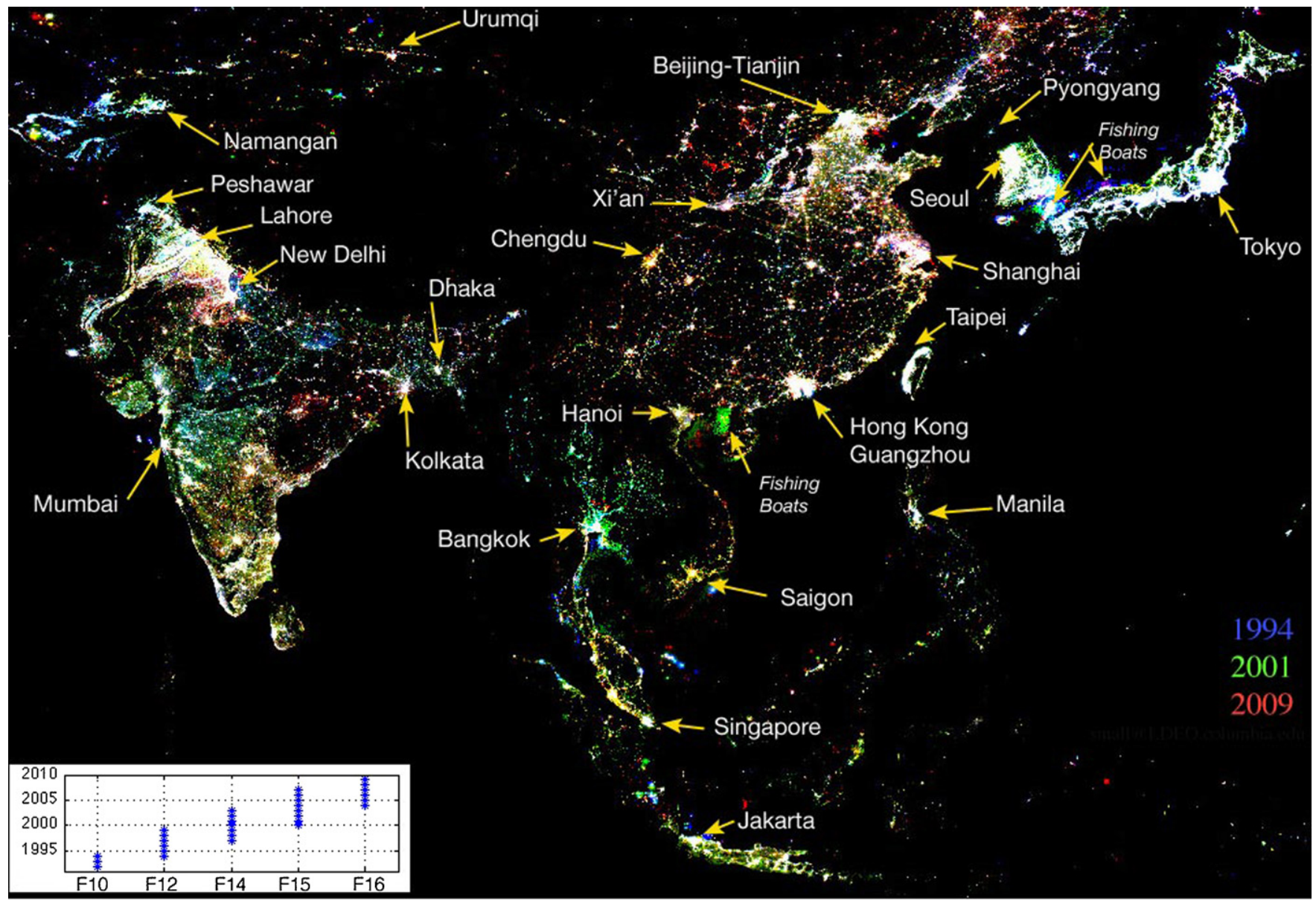

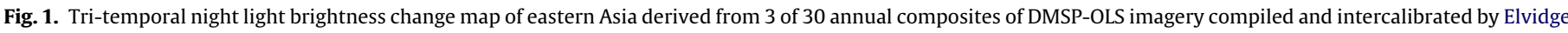

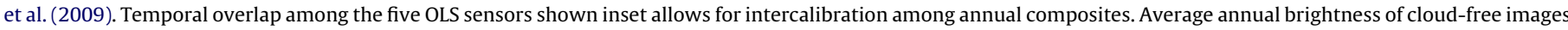

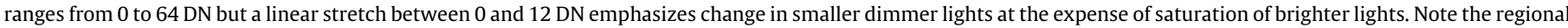

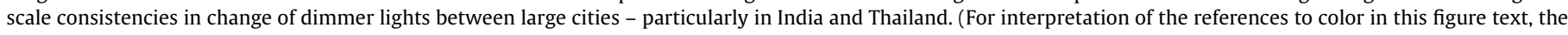
reader is referred to the web version of the article.)

relative contribution of each EOF to the corresponding pixel time series $P_{x t}$ at each location $x$. The relative contribution of each EOF to the total spatio-temporal variance is given by the eigenvalues of the covariance matrix. The distribution of eigenvalues also gives an indication of the dimensionality of the data in terms of uncorrelated modes of variance.

In this study dimensionality refers to the structure of the spatiotemporal patterns represented in the data - and their relative magnitude compared to the stochastic variance. In oceanography and meteorology the PC transformation is often used to characterize dynamically important modes of spatiotemporal variance associated with physical processes. The implicit assumption is that some number, $D(\leq N)$, of the low order EOFs and their corresponding PCs represent deterministic processes and that the higher order dimensions represent stochastic variance $\varepsilon$. This allows an observed pixel time series to be represented as a sum of deterministic and stochastic components as:

$P_{x \mathrm{t}}=\sum_{i=1}^{D} C_{i x} F_{i \mathrm{t}}+\varepsilon$

EOFs are generally spatial patterns intended to represent spatially continuous modes of variability of physical processes while the PCs are generally the weights representing the temporal contribution of the corresponding spatial pattern (Preisendorffer, 1988; von_Storch and Zwiers, 1999). In this study the convention is reversed so the EOFs represent temporal patterns and the PCs represent the corresponding spatial weights. In conventional EOF analysis, there is often an attempt to interpret different EOFs in terms of dynamical processes. However, this can be challenging because the EOFs merely represent statistically uncorrelated modes of variance but are not necessarily dynamically distinct. In the context of this study we consider multiyear interannual to decadal trends to result from deterministic processes and higher frequency year-to-year variability to result primarily from stochastic processes related to actual brightness variations as well as spatial and temporal sampling effects. We acknowledge that some year-toyear variability may actually result from deterministic processes but that it may be effectively indistinguishable from stochastic variability in these data.

In this analysis we show that the low order EOFs of night lights do represent distinct components of change but not necessarily independent processes. The diversity of processes responsible for presumed deterministic changes in night light extent and brightness can be represented in terms of both average brightness and the sense and timing of change. The temporal patterns are given by the EOFs while the relationships among these patterns are given by the topology of the temporal feature space with dimensions defined by the low order PCs. The dimensionality is indicated by the size distribution of the eigenvalues. For this analysis we use the uncentered, unscaled PC transform based on the eigenstructure of the covariance matrix - but the method we present could also use the 
a Asia Lights 1992-2009 Intercalibrated \& Clipped
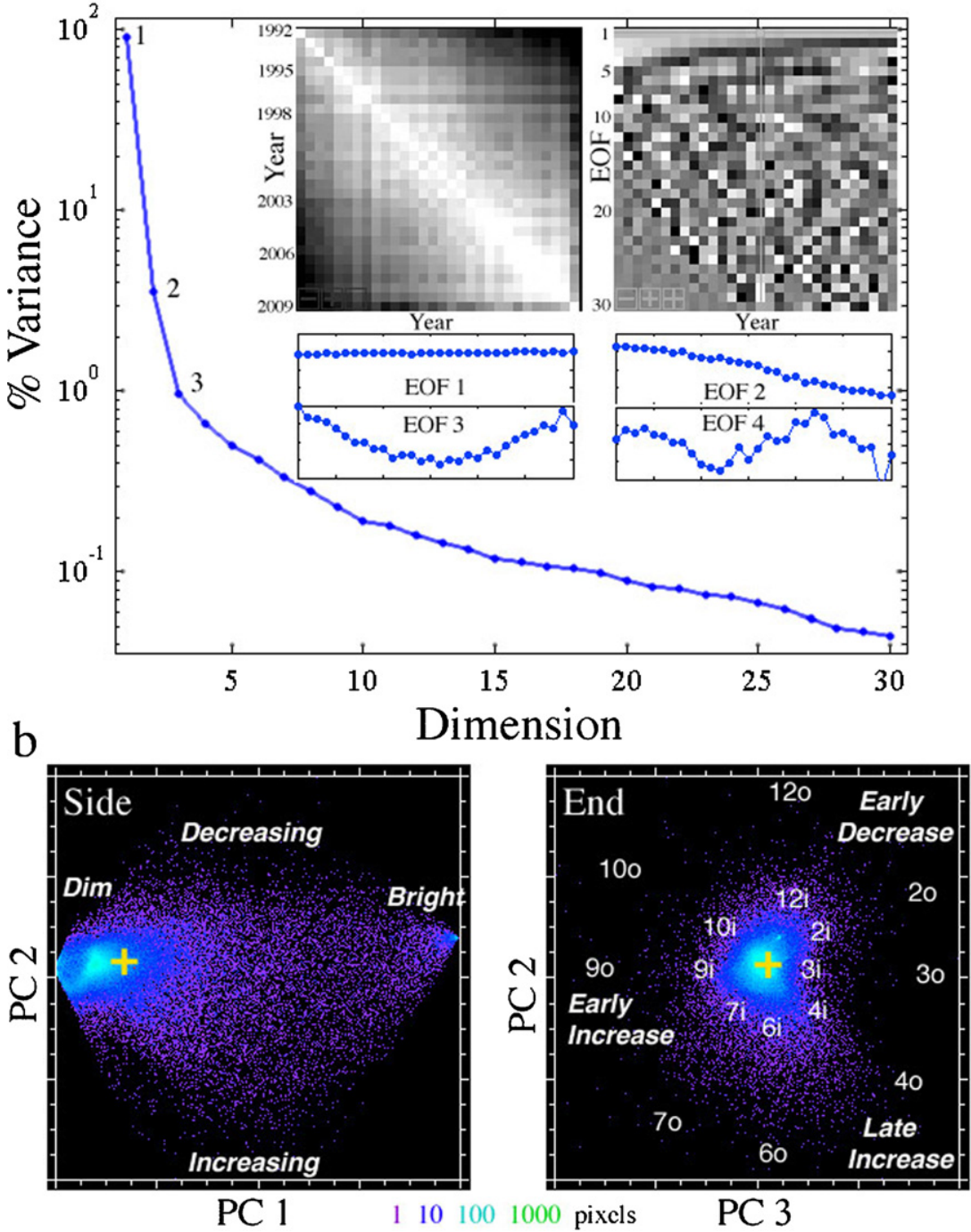

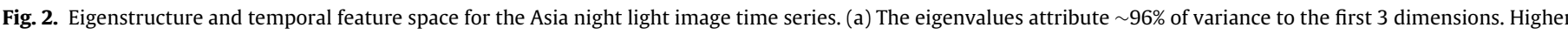

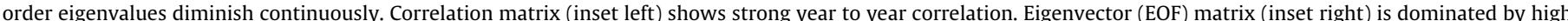

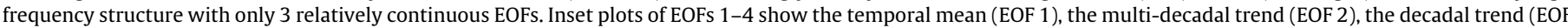

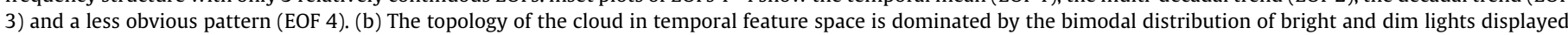

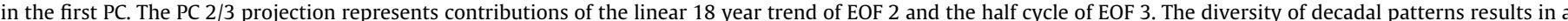
constellation of distinct EOF $2 / 3$ combinations surrounding the bimodal brightness axis in PC1. Inset numbers on end view correspond to time series in Fig. 3 .

transform based on the correlation matrix with or without scaling. Additional details of the approach are given by Small (under review).

\section{Characterizing spatio-temporal change}

We use empirical orthogonal function (EOF) analysis to quantify patterns of temporal change in OLS stable night light brightness and spatial extent over the past 19 years. Intercalibrated data are stacked into a spatial time series of images of annual average brightness resulting from both brightly lit cities and from dimmer, less intensively developed rural areas. Meaningful combinations of EOFs can be derived from the topology of the temporal feature space. The temporal feature space, defined by the low order principal components, represents the spatial distribution of image pixel time series in terms of the relative contribution of the temporal EOFs (Small, under review). The approach used here is based on EOF analyses commonly used to represent geophysical processes in oceanography and meteorology (Storch and Zwiers, 1999) - but casts the EOFs as temporal rather than spatial modes and uses the topology of the low order PC space to determine which linear combinations of low order EOFs depict physically meaningful temporal patterns (Small, under review).

The spatio-temporal variance in the night light data is represented by the eigenstructure of the covariance matrix and the topology of the temporal feature space. These are illustrated in Fig. 2. The eigenvalues show that the first two dimensions account for much more variance than the remaining continuum of dimensions. The structure of the three low order EOFs is also more continuous than the higher order EOFs. The topology of the temporal feature space shows the combination of EOFs that represent most of the spatiotemporal variance in the image time series. Fig. 2 shows the variance structure of the data and the relationship between the spatial PCs and corresponding temporal EOFs in the three low order PC dimensions that represent $91 \%$ of the total variance. The separation of the overall brightness from the decadal changes is apparent in the structure of the temporal feature space. The topology of the feature space is similar to that of 

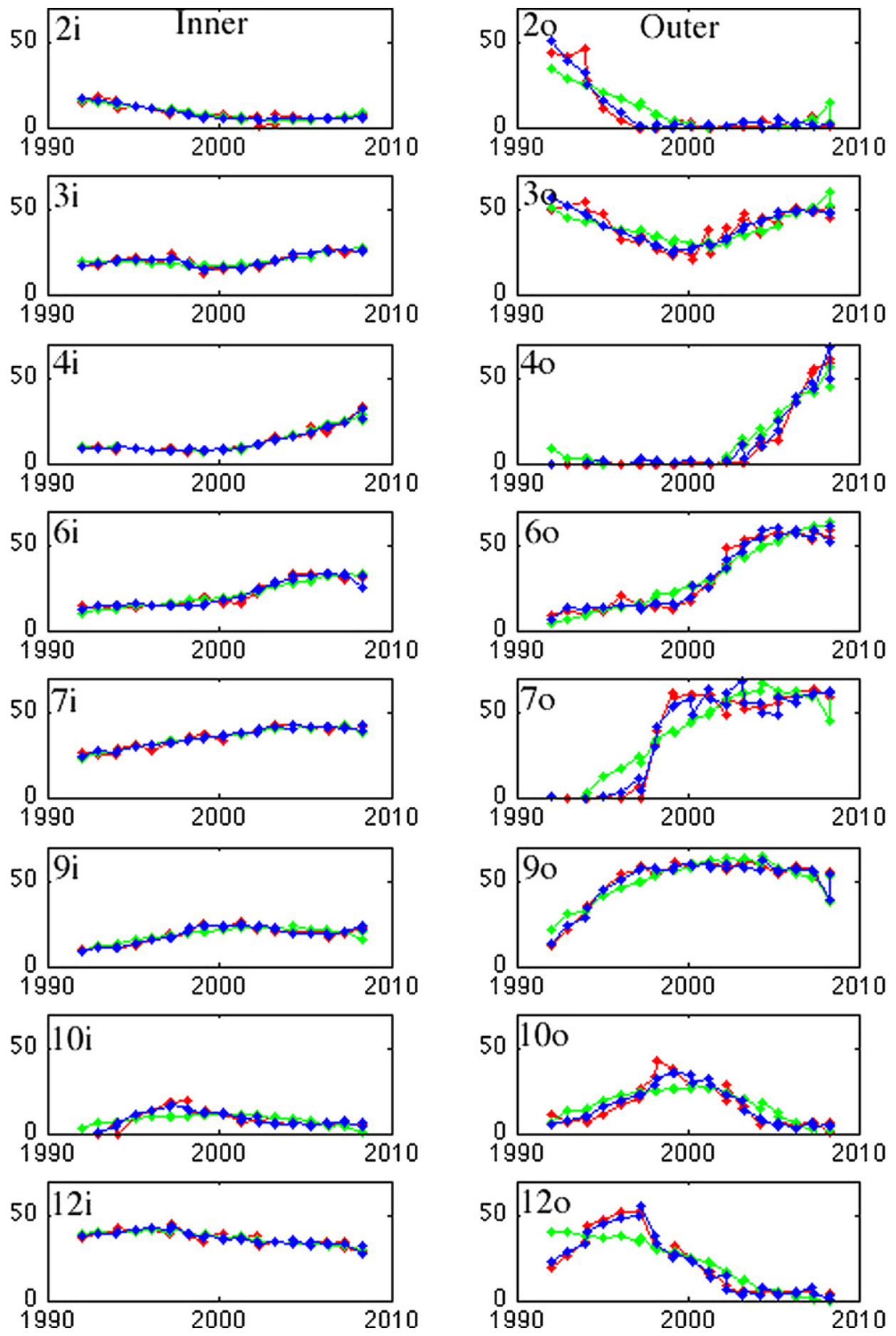

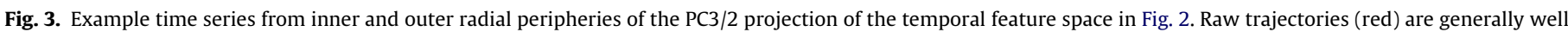

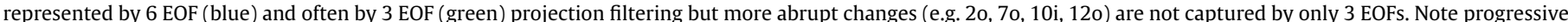
shift of extrema with location in PC $3 / 2$ space. (For interpretation of the references to color in this figure legend, the reader is referred to the web version of the article.)

an HSV color space. In both spaces the brightness forms a central axis about which a circular continuum of temporal changes (peak years $=$ hues) are distributed. This color analogy extends naturally to the tri-temporal change map. When a single year of brightness is represented by a primary color channel $(\mathrm{R}, \mathrm{G}$, or $\mathrm{B})$ the temporal changes in brightness correspond to continuous variations in hue - both in the HSV analogy and in the composite image.

The intercalibration of the annual night light composites considerably reduces spurious interannual variability - but does not eliminate it entirely. Significant year to year variability remains in the intercalibrated image time series. Much of this spurious variance is related to spatial uncertainty in the coregistration of the annual composites. In addition, the phenomenon of low luminance "overglow" results from a combination of limited sensor resolution, atmospheric scattering and subannual spatial coregistration uncertainty in the compositing process. This overglow causes spatial blurring within individual annual composites. Additional temporal blurring results from interannual registration uncertainty. The result is a combination of spurious spatial blurring and temporal variance superimposed on whatever actual changes in brightness 


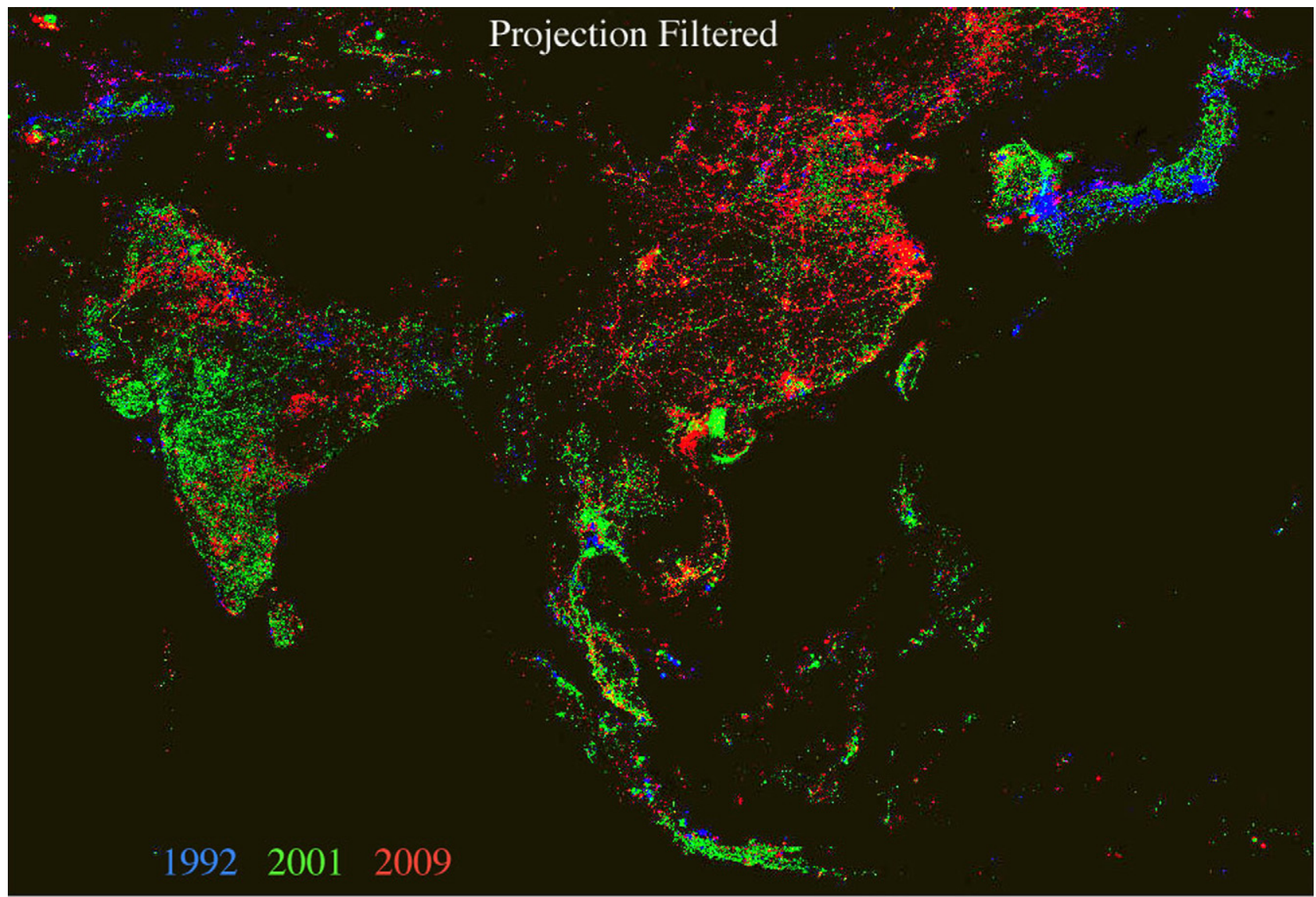

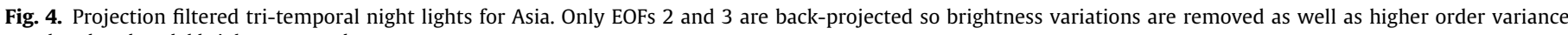
unrelated to decadal brightness trends.

may be measured by the sensor. Spatial or temporal filtering has the undesirable effect of mixing this spatiotemporal noise with the signal in unknown proportions.

We address the spatial blurring and temporal noise problems by using the information contained in the temporal EOFs and their spatial abundance and distribution in the temporal feature space. The projections of the low order PCs reveal the relationship among the low order EOFs in terms of the actual temporal EMs that span the space of all temporal patterns present in the image time series. The variance partition of the PC transform makes use of the spatial abundance of different temporal patterns to distinguish the components of the temporal patterns that the greatest number of pixel time series have in common. Two orthogonal projections of the three low order PCs for the night light time series for southeastern Asia are shown in Fig. 2 - along with the 3 low order EOFs that represent $\sim 96 \%$ of the variance in the image time series. EOF 1 represents the temporal mean brightness of the image time series and accounts for $\sim 91 \%$ of the variance. EOFs 2 and 3 represent the decadal trends that explain the next $5 \%$ of variance. The remaining 27 modes each represent less than $1 \%$ and together less than $4 \%$ of the total variance in the image time series. On the basis of the clear break in the slope of the eigenvalue spectrum and the smoothness of the 3 low order temporal EOFs, we infer that the interannual growth component of the night light data is represented primarily by the first three dimensions and attribute the remaining $4 \%$ of variance in dimensions $>3$ to stochastic and local processes.

The structure of the projection of PCs 2 and 3 highlight the difference between early (1990s) and late (2000s) increases and decreases in the two decade time series. By inverse transforming only dimensions 2 and 3 we can simultaneously remove both the spurious variance and the dominant mean brightness from the time series. This emphasizes only the decadal trends represented by early and late increases and decreases in brightness. In the temporal dimension, the result of this projection filtering is smoothed time series lacking the spurious variance that is not reflected in the low order EOFs depicting the most spatially abundant temporal patterns (Fig. 3 ). The projection filtered image time series does not contain the spurious year-to-year variability and allows the decadal trends of bright and dim lights to be shown simultaneously on the same map. Fig. 4 shows a projection filtered tri-temporal composite of the same years shown in Fig. 1. Note that the saturated bright areas in Fig. 1 are replaced by primary colors emphasizing the decadal trends.

Projection filtering depicts the decadal patterns of change in night light brightness without the distraction of spurious year-toyear differences in apparent brightness and the ten-fold difference in brightness between the bright, heavily developed urban centers and the much dimmer peripheral areas where development and urban growth begins. The tri-temporal change maps presented here use the same display conventions to compare tri-temporal composites of intercalibratted night light with a projection filtered tri-temporal composite of the same annual composites with only the decadal patterns associated with the 2nd and 3rd dimensions of the PCs and EOFs. In both composites warmer colors (red, yellow) indicate brightening over the past 18 years. Cooler colors (blue, cyan) indicate dimming. Green areas show some brightening during the 1990s followed by some dimming in the 2000s; magenta areas have the opposite pattern. The effect of projection filtering on brightness time series is shown in Fig. 4. Inverse transforming 3 low order dimensions represents most decadal trends accurately but smooths some abrupt changes (e.g. 7o) Inverse transforming 6 low order dimensions better represents 

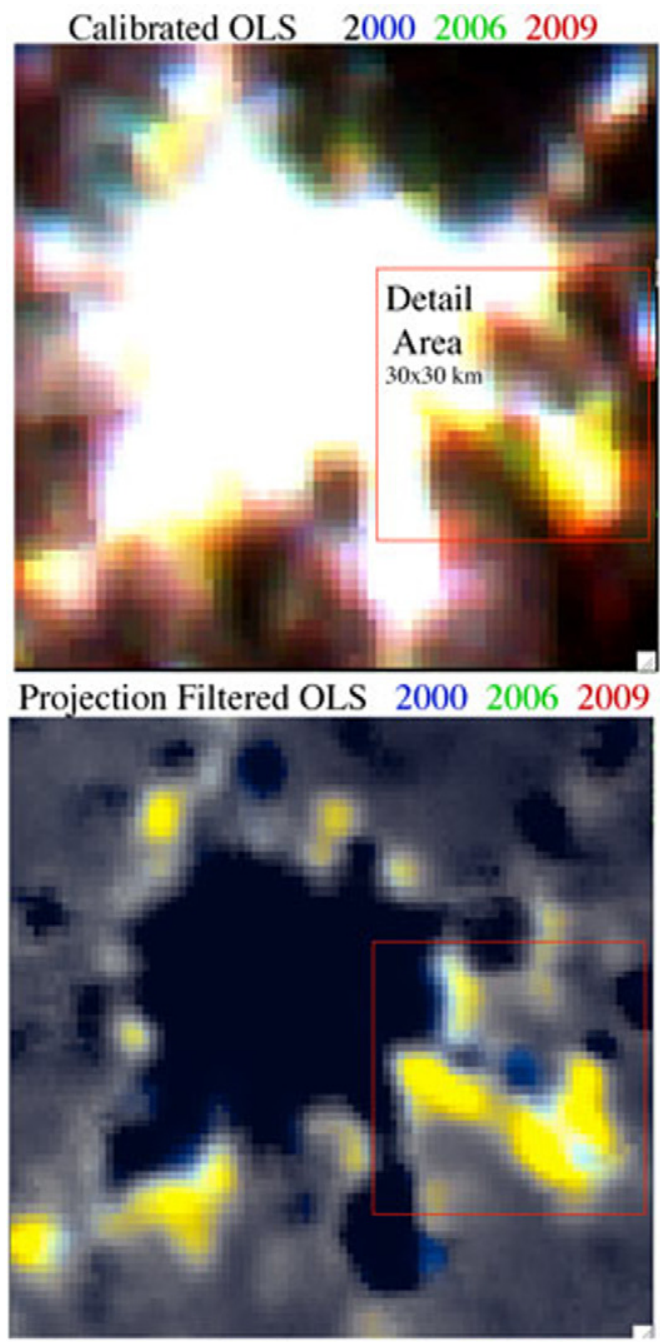

Substrate Fractions 200020062010

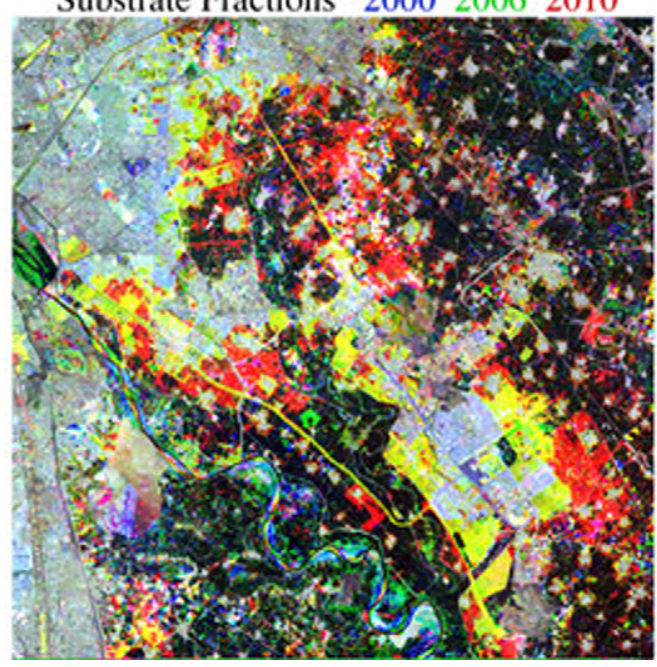

$19 / 2 / 2000$
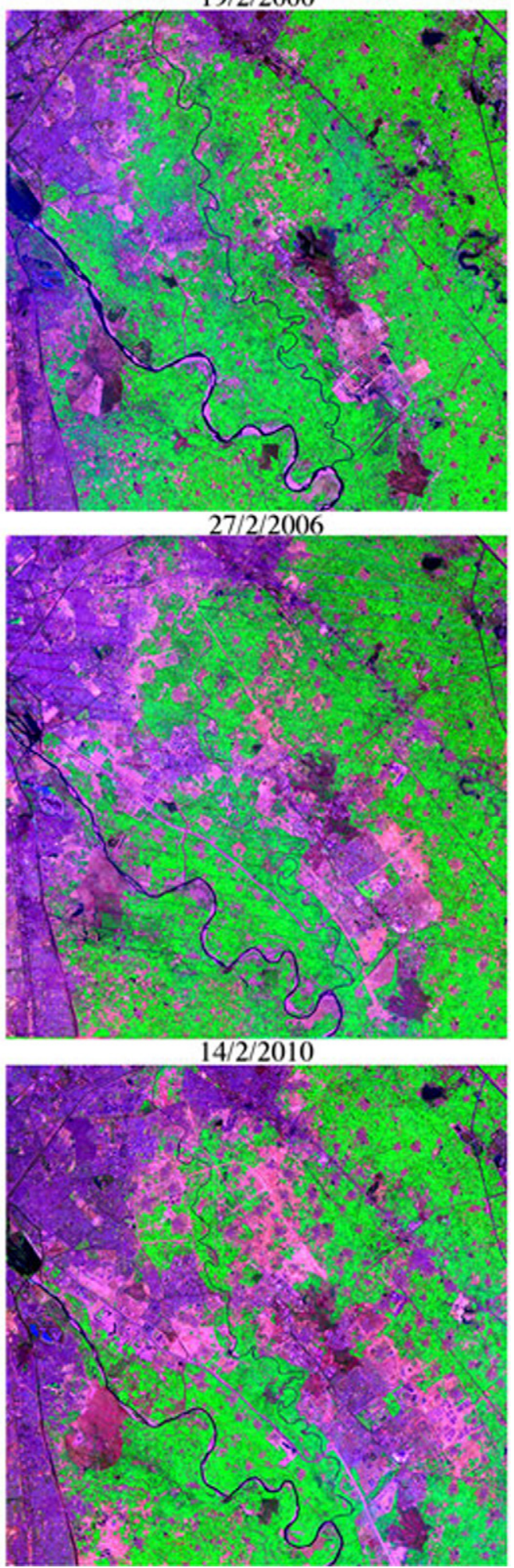

Fig. 5. Tri-temporal Landsat change comparison for New Delhi. Note infilling development, increase in shadow fraction (darkening) and radial road construction.

these abrupt changes while still eliminating spurious year-to-year variance.

\section{Comparisons with Landsat}

Multitemporal Landsat imagery provides independent corroboration of land cover changes suggested by observed changes in night light. In areas with sufficient Landsat coverage we can use changes in land surface reflectance to infer changes in land cover that may correspond to changes in anthropogenic lighting detected by OLS. Here we represent changes in exoatmospheric reflectance in terms of spectral endmember fractions (Small, 2005). Global analyses of Landsat imagery show that $98 \%$ of land surface reflectance can be represented accurately as linear

Please cite this article in press as: Small, C., Elvidge, C.D., Night on Earth: Mapping decadal changes of anthropogenic night light in Asia. Int. J. Appl. Earth Observ. Geoinf. (2012), doi:10.1016/j.jag.2012.02.009 

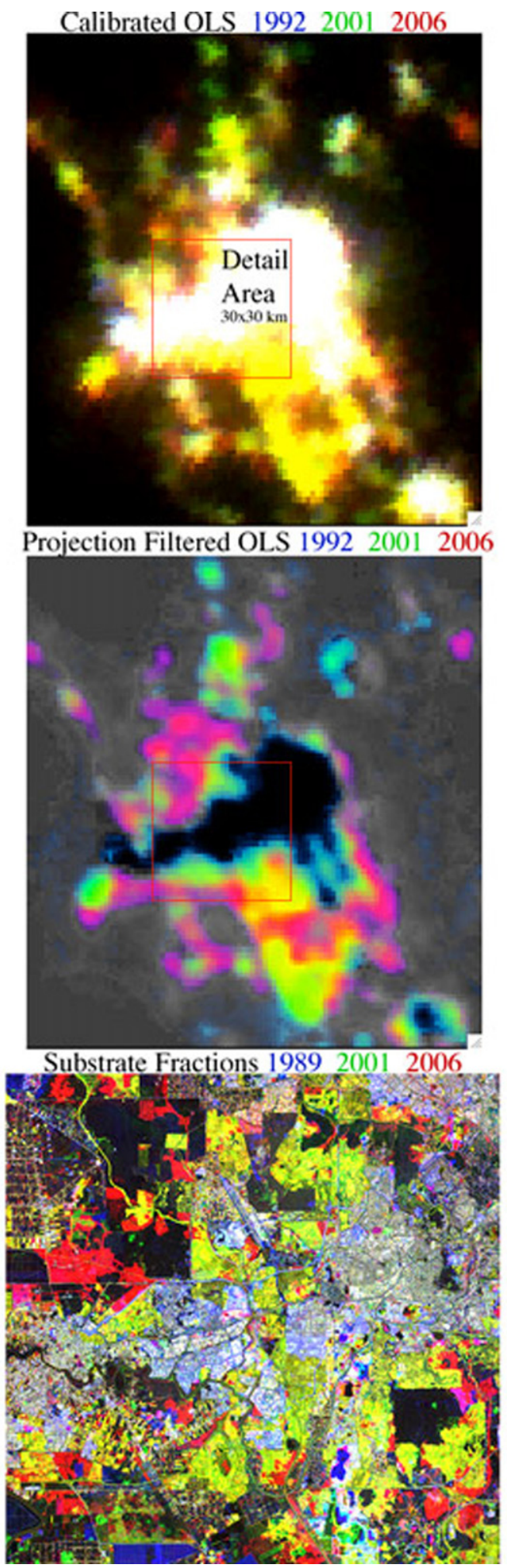
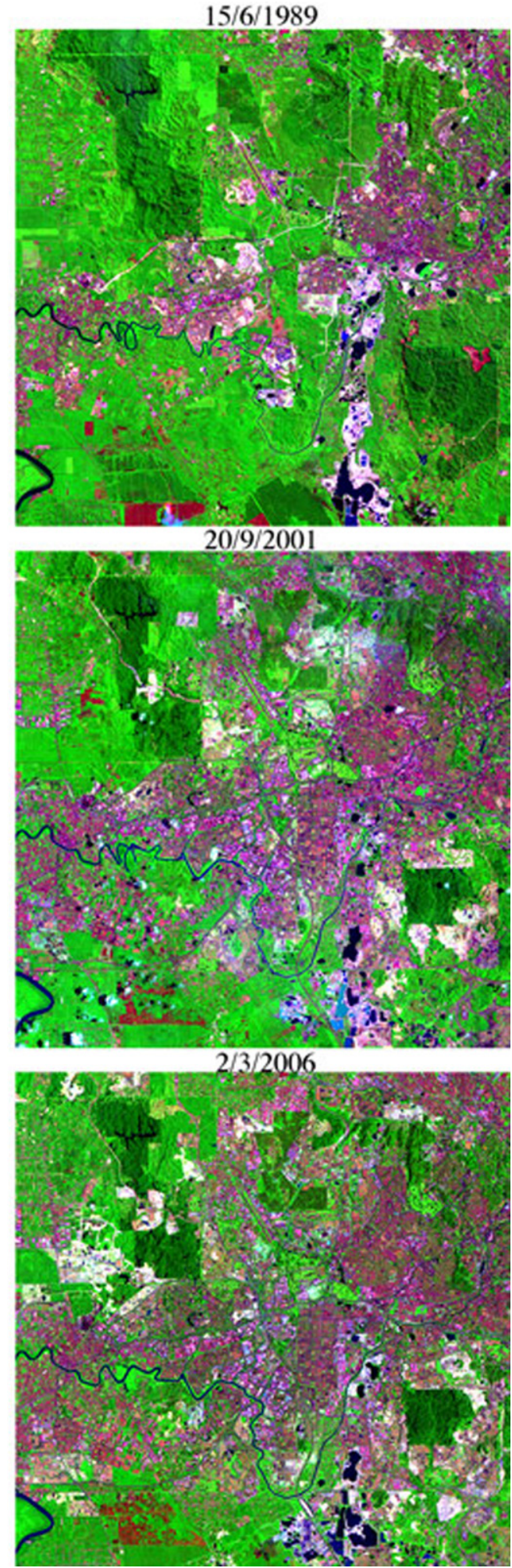

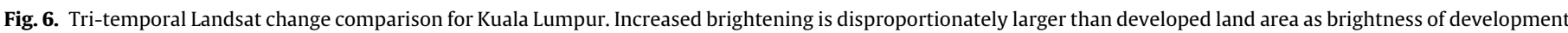
varies.

mixtures of rock-soil substrate, vegetation and dark surface/shadow (Small, 2004). Global analyses of urban reflectance show that most impervious surfaces are spectrally indistinguishable from rock-soil substrates when aggregated spatially and spectrally to Landsat resolutions. In temperate and tropical environments, where undeveloped land is generally vegetated, high substrate fractions usually correspond to anthropogenic development or fallow agricultural areas. To illustrate the correspondence between spatiotemporal changes in land cover and changes in night light we compare tri-temporal maps of Landsat derived substrate fraction to contemporaneous tri-temporal maps of night light.

Tri-temporal night lights reveal a pronounced increase in brightness extending southeastward from New Delhi since 2000 

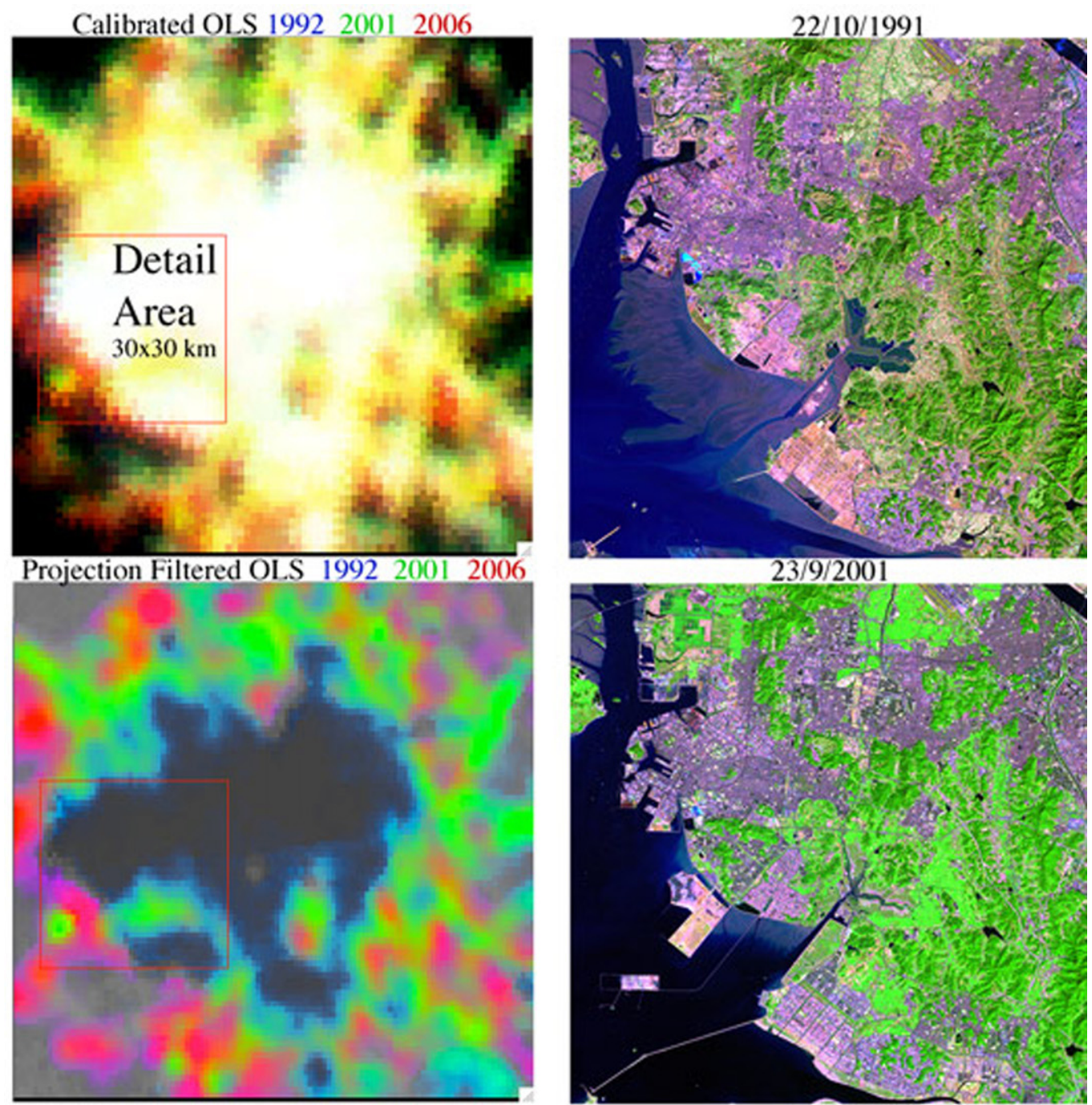

Substrate Fractions 199120012006
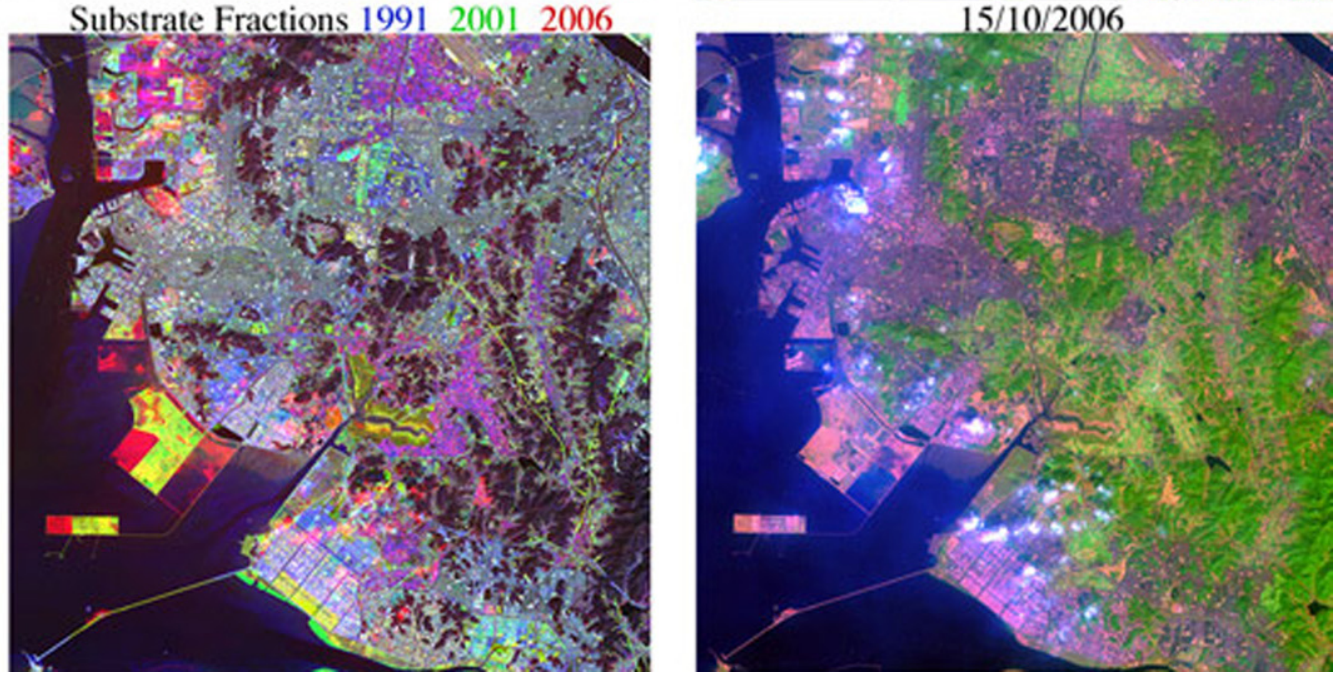

Fig. 7. Tri-temporal Landsat-OLS change comparison for Seoul. Note multiphase reclamation along coast and post 2001 overglow over water.

(Fig. 5). Near-anniversary Landsat images, acquired during the growing season clearly distinguish standing crops form urban development. In most areas urban development takes the form of substrate-shadow mixtures with periodic 2D texture resulting from streets and building shadow. The tri-temporal fraction map shows this expansion as discrete transitions from vegetation to substrate-shadow mixtures. Several long straight thoroughfares also appear during the later interval. It is important to note that the agricultural areas surrounding the development are not completely unlighted. They correspond to low levels of background luminance (DN 5-10) extending well beyond the overglow of the brighter areas.

Tri-temporal night lights reveal a more complex multiphase growth extending outward from a corridor west of Kuala Lumpur

Please cite this article in press as: Small, C., Elvidge, C.D., Night on Earth: Mapping decadal changes of anthropogenic night light in Asia. Int. J. Appl. Earth Observ. Geoinf. (2012), doi:10.1016/j.jag.2012.02.009 


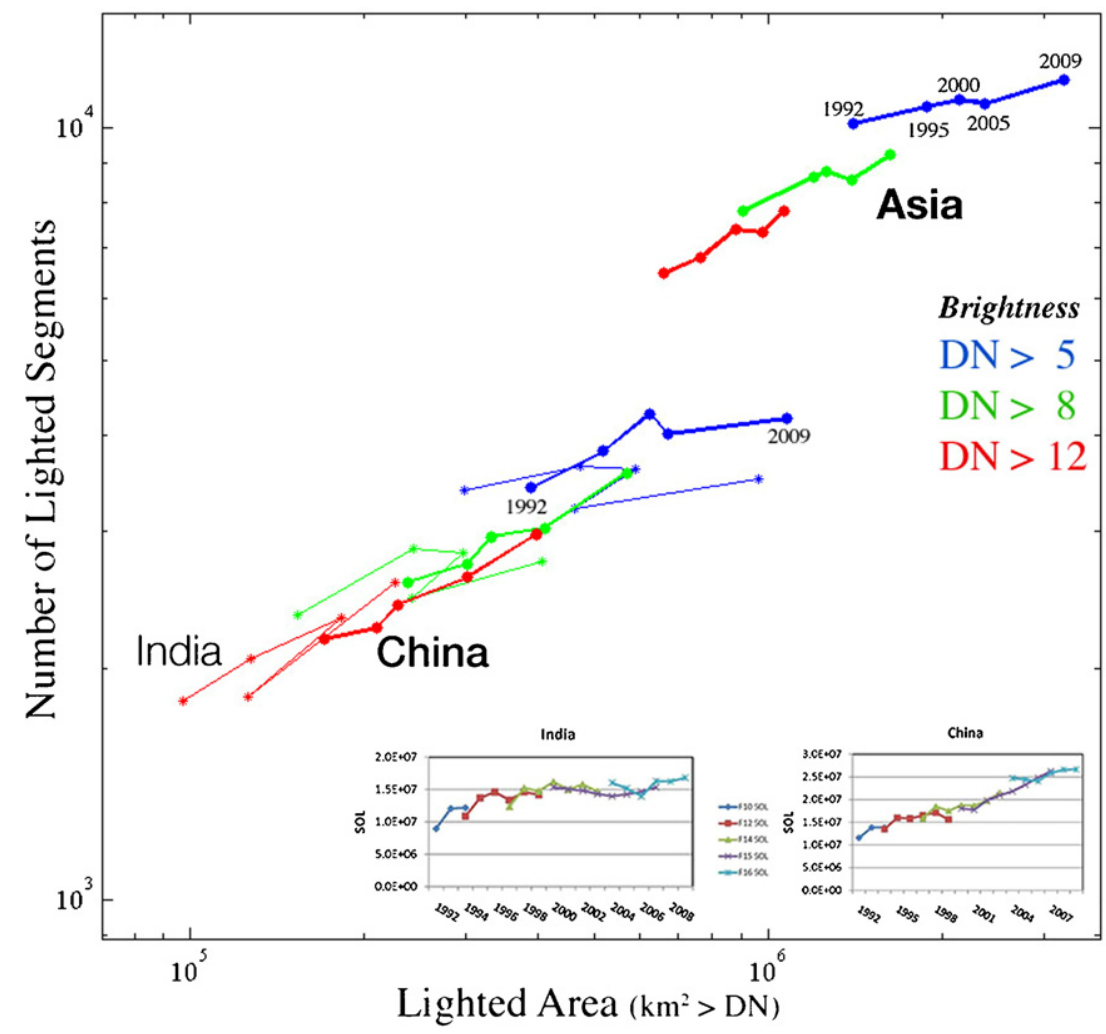

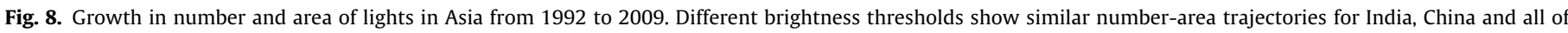

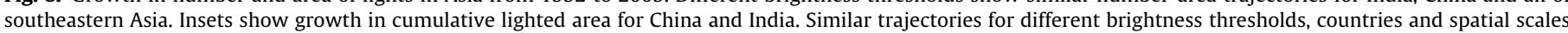
suggest consistency in nucleation and growth processes on decadal time scales.

(Fig. 6). In this case, the tri temporal substrate map shows a less spatially continuous pattern of development with patches of 1990s change interspersed with patches of 2000s change and large areas of unchanging vegetation. Close comparison of the tri-temporal substrate and light composites reveals a disparity between the area extents of land cover and lighting change. This shows that brightness changes are often larger in spatial extent than the corresponding land cover change. The implication is that changes in developed areas may overestimate changes in developed area at fine spatial scales and high brightness levels. For this reason, we do not generally assume that they correspond to equal changes in developed area.

Tri-temporal changes in brightness and extent of night lights in the vicinity of Seoul reveal a very different scenario from the previous two examples (Fig. 7). While the magnitude of brightening around Seoul is comparable to New Delhi and Kuala Lumpur, the spatial patterns shows more infilling and brightening of developed areas than outward growth. The tri-temporal substrate map shows most areas unchanged with some areas of reclamation along the coast. The tri-temporal lights also show a pronounced increase in overglow over water post 2006 suggesting brightening of the developed coastal areas. Another limitation of night light is illustrated by the area of infilling development near the top center of the tri-temoral substrate map. Prior to this development the overglow from the surrounding areas was already saturated so the infilling development is not captured by the night light. Interstitial brightening, not corresponding to land cover change suggests that brightening may occur as a result of increased outdoor lighting in previously developed areas.

These Landsat-OLS comparisons, along with those previously published (Small et al., 2011), illustrate two important caveats to the interpretation of night light imagery - particularly changes in brightness:
1) Changes in brightness correspond to changes in luminance not necessarily changes in developed area. Because overglow is proportional to brightness (Small et al., 2005), increases in brightness are expected to cause increases in overglow in surrounding areas. Therefore, temporal changes in lighted area may result from increases in developed area or increases in intensity of development.

2) Most area of low level luminance ( $<\sim 15$ DN) does not correspond to overglow from large bright cities. Large areas of low level luminance extend over agricultural areas with numerous small settlements but no large cities (see www.LDEO.columbia.edu/ small/DayNight for example comparisons). For this reason, changes in brightness should be considered in the context of the background luminance level rather than absolute change alone. Therefore the projection filtered change map should always be used as a reconnaissance tool in conjunction with the corresponding calibrated tri-temporal change map.

\section{Comparison of changes in number and extent of lights}

Spatiotemporal change in luminance takes two complementary forms: change in the number of spatially distinct lighted areas and change in total lighted area above or below some brightness threshold. Together, these result in changes in the total sum of lights (SoL). These metrics of change represent three different types of change in anthropogenic land cover: nucleation, aggregation and intensification. Fig. 8 shows the simultaneous growth of lighted area and number of lights for China, India and southeastern Asia between 1992 and 2009. These trajectories are shown for three brightness thresholds spanning a critical percolation transition where the spatial connectivity, and therefore size distribution, undergoes explosive percolation and 

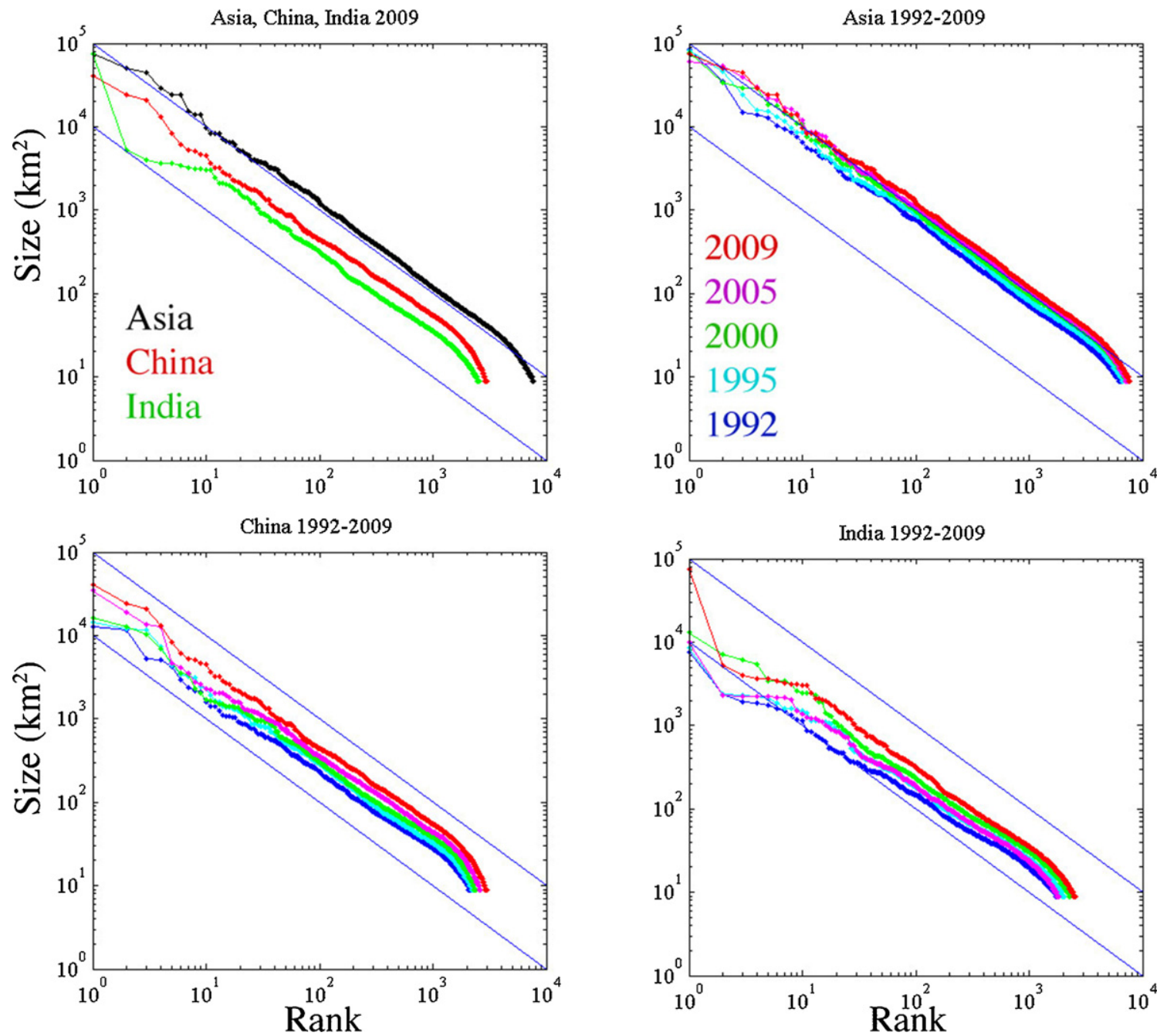

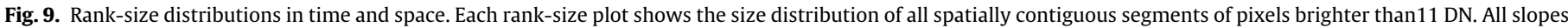

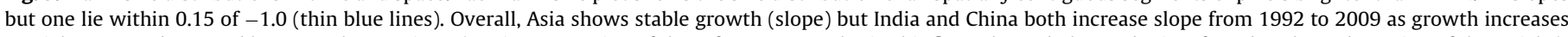

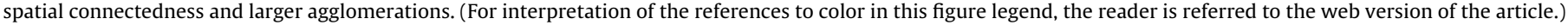

becomes superconnected spatially (Small et al., 2011). Despite different brightness thresholds, spatial scales and geographies, the area-number trajectories (slopes) for China, India and all of southeastern Asia are very similar. This suggests a remarkable consistency in nucleation and growth processes on decadal time scales - despite the very different sizes and growth histories of China and India (Fig. 8, insets).

In spite of the similarities in growth trajectories, there are some important differences between China, India and the whole of southeastern Asia. Even though India and China have similar areas above the low brightness threshold ( $5 \mathrm{DN}$ ), China has nearly twice the area of brighter $(\mathrm{DN}>12)$ lights as India. In 2009, India and China account for $2 \times 10^{5}$ and $4 \times 10^{5}$ (respectively) of southeastern Asia's $\sim 1 \times 10^{6} \mathrm{~km}^{2}$ of area brighter than $12 \mathrm{DN}$. The area-number trajectories for Asia have similar slopes to those of India and China but are displaced to greater numbers of lights for comparable areas of light. This reflects the large number of smaller isolated lights on the archipelagos and coastlines of southeastern Asia compared to the more clustered inland lighted areas in India and China.

Interestingly, some areas in India appear to have lost considerable number and area of lights around 2005. The decreases have little effect on the sum of lights suggesting that they are mostly dimmer lights that do not contribute as much to the total sum of light as they do to the number and area of lights. This apparent change in background luminance, visible in large, spatially continuous regions of India (Fig. 1), could result from either anthropogenic, atmospheric, imaging or compositing processes. At this point we lack sufficient information to determine how each process contributes to the apparent decrease but we point out that both F15 and F16 composites show significant decreases for 2005 and 2006 in spatially contiguous areas of India - but not everywhere.

Both area and number of lights well as sum of lights are aggregate measures that reflect changes in the overall spatial distribution of lighted area. A more complete representation is given by the rank-size distribution of spatially contiguous lighted segments and how this distribution changes in space and time. The ranksize distribution shows all spatially distinct lights sorted by area. Fig. 9 shows rank-size distributions of lighted areas brighter than $\mathrm{DN}=11$ for India, China and all of southeastern Asia. All of the distributions are strongly linear over four orders of magnitude, suggesting a power law or similar heavy tailed distribution. Similar distributions are also obtained for DN thresholds of 9 and 6 - but with correspondingly larger areas. The slope and linearity of the distributions do not change appreciably over this range of thresholds suggesting stability of the underlying distributions. 
Zipf's Law predicts a power law rank-size distribution with a slope of -1 for city size distributions as measured by population (Zipf, 1942, 1949), although the linearity and slope of country level and aggregated population distributions varies considerably (Nitsch, 2005; Soo, 2005). While the consistency of Zipf's Law has attracted sustained interest for several decades, and has been the basis for a multitude of models (e.g. Berry and Garrison, 1958; Gabaix, 1999; Lotka, 1941; Pumain, 2006), the varying degree and extent of agreement with observation impede consensus on either the universality of the law (Gan et al., 2006; Krugman, 1996; Soo, 2005) or its underlying causes (Batty, 2006; Lotka, 1941; Pumain, 2004, 2006). The assertion of a universal power law for city size (as represented by population) is controversial because the estimates of linearity and slope of the power law rank-size distribution vary over time and among countries (Gabaix et al., 2004; Nitsch, 2005; Pumain and Moriconi-Ebrard, 1997; Soo, 2005).

Despite mixed results obtained from census enumerations, the connectivity and size distribution of lighted areas in Asia seems to be consistent with Zipf's Law in both space and time. The slope of all but one of the distributions for brightness thresholds of 6,9 and $12 \mathrm{DN}$ are within 0.15 of -1 (Fig. 9). This is consistent with recent global and continental scale analyses of area distributions of both population density and night light brightness which are well fit by power laws with slopes near -1 for a wide range of thresholds (Small et al., 2011). As in the global and continental scale analyses, the largest spatially contiguous segments in southeastern Asia are much larger than the individual cities. At scales of $10^{4}$ to $10^{5} \mathrm{~km}^{2}$, these large agglomerations correspond to enormous spatial networks of interconnected cities and smaller settlements with interstitial areas of lower population or brightness. Yet the size distributions still conform remarkably well to the power law ranksize distribution predicted by Zipf's Law for individual settlements defined by population.

The temporal consistency of rank-size slope for India, China and southeastern Asia through time suggests relatively stable growth throughout the size range over nearly two decades - albeit at very different rates. The rank-size distribution for India falls proportionately at all sizes in 2005 as expected. Together with the consistent trajectory of number area plots in Fig. 8, this suggests that aggregate growth processes seem to be quite robust across a range of areas, geographies and thresholds through time. In spite of this consistency, some temporal increases in the rank-size distribution suggest that spatial aggregation is exceeding nucleation in India and China - but not overall. This apparent increase is analyzed in greater detail in a separate study.

\section{Conclusions and implications}

Decadal changes in night light brightness, identified by EOF analysis, form coherent spatial patterns at local and regional scales. In areas where sufficient Landsat coverage exists these changes in brightness correspond to areas where land cover change suggests expansion of anthropogenic land use. The implication is that multi-temporal night lights can provide a global proxy for lighted anthropogenic development.

Over the past two decades, consistency in the increase in night lights in Asia suggest consistency in the underlying processes of growth and development. Rank-size distributions of spatially contiguous night lights in India, China and throughout southeastern Asia show strongly linear trends with slopes near -1 for a range of brightness thresholds. While slopes persist near -1 through time for southeastern Asia, increasing sub-unity slopes for India and China suggest that development in these countries is resulting in increased connectivity of the larger spatial networks of development at the expense of nucleation of smaller isolated settlements. Taken together with the relatively stable near-unity slopes of rank-size distributions for southeastern Asia these results suggest considerable spatial variations in the growth processes contributing to an apparently stable distribution at continental scales. The implication is that the logarithmically uniform distribution observed by Zipf and others may be an emergent phenomenon arising from spatial aggregation of non-uniform distributions at finer spatial scales.

\section{Acknowledgment}

This work was funded by the United States National Institutes of Child Health and Development [award R21 HD054846] to CS.

\section{References}

Adams, J.B., Smith, M.O., Johnson, P.E., 1986. Spectral mixture modeling; a new analysis of rock and soil types at the Viking Lander 1 site. Journal of Geophysical Research 91, 8098-8122.

Batty, M., 2006. Heirarchy in cities and city systems. In: Pumain, D. (Ed.), Hierarchy in Natural and Social Sciences. Kluwer, Dordrecht, Germany, pp. 143-168.

Baugh, K., Elvidge, C., Ghosh, T., Ziskin, D., 2009. Development of a 2009 stable lights product using DMSP-OLS data. In: Elvidge, C. (Ed.), Proceedings of the 30th AsiaPacific Advanced Network Meeting. Hanoi, Vietnam, pp. 114-130.

Berry, B.J.L., Garrison, W.L., 1958. Alternate explanations of urban rank-size relationships. Annals of the Association of American Geographers 1, 83-91.

Bretherton, C.S., Smith, C., Wallace, J.M., 1992. An intercomparison of methods for finding coupled patterns in climate data. Journal of Climate 5, 541-560.

Briggs, D.J., Gulliver, J., Fecht, D., Vienneau, D.M., 2007. Dasymetric modeling of small-area population distribution using land cover and light emissions data. Remote Sensing of Environment 108, 451-466.

Byrne, G.F., Crapper, P.F., Mayo, K.K., 1980. Monitoring land-cover change by principal component analysis of mulittemporal Landsat data. Remote Sensing of Environment 10, 175-184.

Crist, E.P., Cicone, R.C., 1984. A physically based transformation of thematic mapper data - the TM tasseled cap. I.E.E.E. Transactions on Geoscience and Remote Sensing GE-22, 256-263.

Doll, C.N.H., Muller, J.-P., Morley, J.G., 2006. Mapping regional economic activity from night-time light satellite imagery. Ecological Economics 57, 75-92.

Eastman, J.R., Fulk, M., 1993. Long sequence time series evaluation using standardized principal components. Photogrammetric Engineering and Remote Sensing 59, 1307-1312.

Elvidge, C.D., Baugh, K.E., Kihn, E.A., Kroehl, H.W., Davis, E.R., 1997. Mapping city lights with nighttime data from the DMSP operational linescan system. Photogrammetric Engineering and Remote Sensing 63, 727-734.

Elvidge, C.D., Tuttle, B.T., Sutton, P.C., Baugh, K.E., Howard, A.T., Milesi, C., Bhaduri, B.L., Nemani, R., 2007. Global distribution and density of constructed impervious surfaces. Sensors 7, 1962-1979.

Elvidge, C.D., Ziskin, D., Baugh, K.E., Tuttle, B.T., Ghosh, T., 2009. A fifteen year record of global natural gas flaring derived from satellite data. Energies 2, 595-622.

Elvidge et al. Remote Sensing of Environment, submitted for publication, http://www.mdpi.com/journal/remotesensing.

Fung, T., LeDrew, E., 1987. Application of principal components analysis to change detection. Photogrammetric Engineering and Remote Sensing 53, 1649-1658.

Gabaix, X., 1999. Zipf's law for cities: an explanation. Quarterly Journal of Economics $114,739-767$.

Gabaix, X., Ioannides, Y.M., Henderson, J.V., Jacques-FranÁois, T., 2004. The Evolution of City Size Distributions. Handbook of Regional and Urban Economics. Elsevier, 2341-2378,Ch. 53.

Gan, L., Li, D., Song, S., 2006. Is the Zipf law spurious in explaining city-size distributions? Economics Letters 92, 256-262.

Ghosh, T., Powell, R., Elvidge, C.D., Baugh, K.E., Sutton, P.C., Anderson, S., 2010. Shedding light on the global distribution of economic activity. The Open Geography Journal 3, 148-161.

Green, A.A., Berman, M., Switzer, P., Craig, M.D., 1988. A transformation for ordering mutispecral data in terms of image quality with implications for noise removal. I.E.E.E. Transactions on Geoscience and Remote Sensing 26, 65-74.

Henderson, J.V., Storeygard, A., Weil, D.N., 2009. Measuring economic growth from outer space. In: National Bureau of Economic Research Working Paper, NBER, Cambridge, MA

Johnson, P.E., Smith, M.O., Adams, J.B., 1985. Quantitative analysis of planetary reflectance spectra with principal components analysis. Journal of Geophysical Research 90, C805-C810.

Kauth, R.J., Thomas, G.S., 1976. The tasseled cap - a graphic description of the spectral-temporal development of agricultural crops as seen by Landsat. In: Proceedings of the Symposium on Machine Porcessing of Remotely Sensed Data, Purdue University, West Lafayette, Indiana, pp. 4B41-44B51.

Krugman, P., 1996. Confronting the mystery of urban hierarchy. Journal of the Japanese and International Economies 10, 399-418. 
Lee, J.B., Woodyatt, A.S., Berman, M., 1990. Enhancement of high specral resoltuion remote sensing data by a noise-adjusted principal components tranform. I.E.E.E. Transactions on Geoscience and Remote Sensing 28, 295-304.

Lotka, A., 1941. The law of urban concentration. Science 94, 164.

Nitsch, V., 2005. Zipf zipped. Journal of Urban Economics 57, 86-100.

Preisendorffer, R.W., 1988. Principal Component Analysis in Meteorology and Oceanography. Elsevier, Amsterdam.

Pumain, D., 2004. Scaling Laws and Urban Systems. Santa Fe Institute, Santa Fe, New Mexico, p. 26.

Pumain, D., 2006. Alternative explanations of heirarchical differentiation in urban systems. In: Pumain, D. (Ed.), Hierarchy in Natural and Social Sciences. Kluwer, Dordrecht, Germany, pp. 169-222.

Pumain, D., Moriconi-Ebrard, F., 1997. City size distributions and metropolisation. Geojournal 43, 307-314.

Richards, J.A., 1984. Thematic mapping from multitemporal image data using the principal components transformation. Remote Sensing of Environment 16, 35-46.

Singh, A., Harrison, A., 1985. Standardized principal components. International Journal of Remote Sensing 6, 883-896.

Small, C., 2004. The Landsat ETM+ spectral mixing space. Remote Sensing of Environment 93, 1-17.

Small, C., 2005. Global analysis of urban reflectance. International Journal of Remote Sensing 26, 661-681.

Small, C., under review. Spatio-temporal dimensionality and time-space characterization of multitemporal imagery. Remote Sensing of Environment.
Small, C., Elvidge, C.D., Balk, D., Montgomery, M., 2011. Spatial scaling of stable night lights. Remote Sensing of Environment 115, 269-280.

Small, C., Pozzi, F., Elvidge, C.D., 2005. Spatial analysis of global urban extent from DMSP-OLS night lights. Remote Sensing of Environment 96, 277-291.

Smith, M.O., Johnson, P.E., Adams, J.B., 1985. Quantitative determination of mineral types and abundances from reflectance spectra using principal component analysis. Journal of Geophysical Research 90, 792-804.

Soo, K.T., 2005. Zipf's Law for cities: a cross-country investigation. Regional Science and Urban Economics 35, 239-263.

Storch, H.V., Zwiers, F.W., 1999. Statistical Analysis in Climate Research. Cambridge University Press.

Sutton, P., Roberts, D., Elvidge, C., Baugh, K., 2001. Census from Heaven: an estimate of the global human population using night-time satellite imagery. International Journal of Remote Sensing 22, 3061-3076.

Townshend, J.R.G., Goff, T.E., Tucker, C.J., 1985. Multitemporal dimensionality of images of normalized difference vegetation index at continental scales. I.E.E.E. Transactions on Geoscience and Remote Sensing GE-23, 888-895.

von_Storch, H., Zwiers, F.W., 1999. Statistical Analysis in Climate Research. Cambridge University Press, Cambridge, UK.

Zipf, G.K., 1942. The unity of nature, least-action, and natural social science. Sociometry $5,48-62$.

Zipf, G.K., 1949. Human Behavior and the Principle of Least-Effort. Addison Wesley. 